Manuscript published in the journal ñColloids and surfaces B: Biointerfacesò vol. 119, 30-37 (2014); PMID: 24859051, doi: 10.1016/j.colsurfb.2014.04.016

\title{
Cationic vesicles based on non-ionic surfactant and synthetic aminolipids mediate delivery of antisense oligonucleotides into mammalian cells
}

Santiago Grijalvo, ${ }^{1}$ Adele Alagia, ${ }^{1}$ Gustavo Puras, ${ }^{2}$ Jon Zárate, ${ }^{2}$ Jose Luis Pedraz, ${ }^{2}$ and Ramon Eritja $^{a^{*}}$

Dedicated to the Memory of Dr. Francisco Sánchez-Baeza and Dr. Nuria Azemar

${ }^{1}$ Institute for Advanced Chemistry of Catalonia (IQAC-CSIC), Department of Chemical and Biomolecular Nanotechnology and Networking Research Centre of Bioengineering, Biomaterials and Nanomedicine (CIBER-BBN), Barcelona, Spain

${ }^{2}$ NanoBioCel group, University of the Basque Country (EHU-UPV), Vitoria and Networking Research Centre of Bioengineering, Biomaterials and Nanomedicine (CIBER-BBN)

*Corresponding author: Institute for Advanced Chemistry of Catalonia (IQAC-CSIC), Jordi Girona 18-26, E-08034-Barcelona, Spain. Tel.: +34934006100.

E-mail address: recgma@ @id.csic.es (Dr. Ramon Eritja). 


\begin{abstract}
A formulation based on a synthetic aminolipid containing a double-tailed with two saturated alkyl chains along with a non-ionic surfactant polysorbate- 80 has been used to form lipoplexes with an antisense oligonucleotide capable of inhibiting the expression of Renilla luciferase mRNA. The resultant lipoplexes were characterized in terms of morphology, zeta potential, average size, stability and electrophoretic shift assay. The lipoplexes did not show any cytotoxicity in cell culture up to $150 \mathrm{mM}$ concentration. The gene inhibition studies demonstrated that synthetic cationic vesicles based on non-ionic surfactant and the appropriate aminolipid play an important role in enhancing cellular uptake of antisense oligonucleotides obtaining promising results and efficiencies comparable to commercially available cationic lipids in cultured mammalian cells. Based on these results, this amino lipid moiety could be considered as starting point for the synthesis of novel cationic lipids to obtain potential nonviral carriers for antisense and RNA interference therapies.
\end{abstract}




\section{Keywords}

Cationic lipids, cationic vesicles, non-ionic surfactant vesicles, antisense oligonucleotide, antisense therapy, gene delivery 


\section{Introduction}

The discovery of antisense technology and more recently RNA interference has allowed new strategies in the search of novel therapeutics by controlling gene expression [1]. These approaches incorporate different action mechanisms than those used in conventional therapies. For this reason the use of nucleic acids may provide enormous benefits for therapy since they inhibit target proteins drugs with high specificity and also become potential units in the treatment of genetic disordered diseases or even in cancer [2].

However, there are many obstacles in developing nucleic acids into therapeutics since they are polyanionic macromolecules. Fortunately, chemical modifications to nucleic acid backbones and/or sugars have accelerated the discovery process of new compounds in addition to improving the properties of nucleic acids in terms of stability against nucleases [3] and decreasing off-targets effects [4] without losing their initial biological activities. Nevertheless, delivery problems continue to be the major bottleneck in the development of nucleic acids as drugs.

Although viral vectors like retroviruses or adenoviruses have shown high transfection efficiencies and have been used in some clinical trials [5] there are concerns about the immunogenicity or the recombination of oncogenes that have still not been solved. Alternatively, non-viral vectors such as lipids [6], cell-penetrating peptides [7], polymers [8] or gold nanoparticles [9] have emerged as promising alternatives to safely delivering nucleic acids.

There are two strategies used for transfecting nucleic acids with non-viral vectors. The first one is the use of formulations [10] which are the simplest and the fastest way to bind non-viral vectors to nucleic acids by taking advantage of the electrostatic interactions between them. The second strategy is the use of covalent approaches in which non-viral carriers and nucleic acids are covalently linked obtaining stable nucleic acid conjugates which improve their biological properties in both in vitro and in vivo [11, 12]. 
There have been great advances made in the last few years in the search for both nucleic acid conjugates as well as formulations for generating active complexes. In addition, there has been renewed interest in the development of new, more efficient and less toxic formulations for nucleic acid delivery.

Since the first transfection experiments carried out by Felgner [13] demonstrated an efficient lipid mediated DNA-transfection by using DOTMA as a cationic lipid, a variety of synthetic cationic lipids have been widely used in formulation in order to deliver therapeutic biomolecules which are becoming promising non-viral tools for nucleic acid delivery [14]. One of the factors that must be considered when using cationic lipids is the tendency of positively charged particles to interact with plasma proteins which induce aggregation and consequently produce low transfection efficiencies in gene delivery [15]. The reduction of the net cationic charge of cationic lipids in formulation, the presence of either serum-resistant cationic lipids [16], an increase of lipid/DNA charge ratios [17], PEGylation [18] and finally the addition of helper lipids into formulations [19] are essential modifications which minimize undesirable effects like cell toxicity in cells and avoiding rapid plasma clearance. Despite efforts made in the development of new formulations or designing novel cationic lipids, obtaining effective non-viral carriers remains crucial for optimal gene transfection.

The use of surfactant agents in colloidal carrier systems might mask or reduce the undesirable effects of cationic lipids. In addition, surfactants may also play an important role in gene delivery $[20,21]$ because the resulting complexes show a high stability. Moreover, their synthesis is readily scalable and the structure is comparable to liposomes. However, there are few studies that have analyzed the effects of such surfactants agents in gene delivery processes $[22,23,24]$. In an effort to develop new formulations based on non-ionic surfactant vesicles, we have recently reported the use of a novel formulation which is composed of a mixture of nonionic surfactant polysorbate- 80 and a synthetic aminolipid containing a double-tailed hydrocarbonated alkyl chain. This demonstrated the ability to efficiently deliver plasmid DNA 
into the retina with good efficiency and low toxicity [25]. In addition, the use of cationic niosome formulations based on the same cationic aminolipid moiety, polysorbate- 80 and squalene were also condensed using plasmid DNA which obtained the corresponding complexes and mediated delivery in several cell lines with high efficiencies [26]. These results prompted us to further investigate the versatility of the aforementioned surfactant formulations in order to encapsulate oligonucleotides and evaluate their effectiveness as a drug delivery system in antisense therapy.

There are few reports in the literature describing the encapsulation of oligonucleotides with nonionic surfactant vesicles [27] which use (in the majority of formulations) a mixture of commercially available cationic lipids which deliver oligonucleotides into cells. Herein, we describe a formulation based on of non-ionic synthetic surfactant vesicles which contain a modified synthetic cationic lipid and an antisense oligonucleotide (ASO) which is designed to knockdown the expression of a Renilla Luciferase gene.

The resulting lipoplexes were fully characterized in terms of zeta potential, average size, stability and electrophoretic shift assay. Finally, the best compositions were used to study-the potential toxicity and transfection processes of antisense oligonucleotides mediated by surfactant vesicles. These results were compared to transfections that were carried out using commercially available cationic lipids.

\section{Materials and Methods}

\subsection{Materials}

All reagents employed in this work were used as received, having an analytical grade and used without further purification. Polysorbate-80 (Tween-80) and 3-(4,5- dimethylthiazol-2-yl)-2,5diphenyltetrazolium bromide (MTT reagent) were purchased from Sigma-Aldrich. Antisense 
phosphorothioate oligonucleotide (sequence 5ôCGT TTC CTT TGT TCT GGA-3ô) complementary to the mRNA of the Renilla luciferase gene targeted to a predominant accessible site between 20 and $40 \mathrm{nt}$ was purchased from Proligo (Sigma-Aldrich). A 18-mer scrambled antisense oligonucleotide sequence (sequence 5ô CTG TCT GAC GTT CTT TGT-3ô was synthesized in-house and purified according to well-established methods (DMTon-based protocols). All the standard phosphoramidites and ancillary reagents used for oligonucleotide synthesis were purchased from Applied Biosystems or Link Technologies. The synthetic aminolipid, 2,3-di(tetradecyloxy)propan-1-amine was obtained as described in the literature [28]. Lipofectamine 2000 was purchased from Invitrogen. PBS buffer and Dulbeccô̂ Modified Eagleôs Medium (DMEM) which was supplemented with a $10 \%$ heat-inactive fetal serum bovine (FBS) along with distilled water (DNAse/RNAse free) were purchased from Gibco. Additional nuclease-free water was also prepared by using $0.1 \%$ of diethylpyrocarbonate (DEPC) to ensure the removal of RNase contamination, autoclaved and filtered before using. Luciferase assay kits were purchased from Promega. Qiagen Giga plasmid purification kit was purchased from Qiagen.

2.2 Preparation of synthetic non-ionic surfactant vesicles with an antisense oligonucleotide (ASO) containing lipoplexes

Non-ionic synthetic surfactant vesicles were prepared using a hydration method with equimolecular amounts of both the synthetic aminolipid and the non-ionic surfactant polysorbate-80. Specifically, equimolecular amounts containing the cationic lipid and polysorbate-80 $(6.40 \mu \mathrm{mol})$ were dissolved in $1 \mathrm{~mL}$ of chloroform. The solvent was evaporated and the resulting crude was kept under vacuum overnight at room temperature. The dried lipid film was hydrated with $1 \mathrm{~mL}$ of sterile HEPES (20 nM; pH 7.4) buffer, filtered previous to use through a $0.2 \mu \mathrm{m}$ membrane filter, and heated to $60{ }^{\circ} \mathrm{C}$ for 20 minutes. The dispersion was vortexed and sonicated for 3 minutes before being used. 
The dispersion was resuspended in HEPES buffer at stock concentrations of $0.484 \mathrm{mM}$ or 828 $\mu \mathrm{M}$ vortexed and was sonicated (3 minutes). Cationic surfactant vesicle/antisense oligonucleotide complexes (lipoplexes) were then formed by adding the required amount of cationic lipid dispersion to aliquots containing fixed amounts of either antisense oligonucleotide targeting the Renilla luciferase mRNA expression or a scrambled oligonucleotide at 14:1 or 16:1 charge ratios ([cationic amino groups $]_{\text {cationic lipid }} /[\text { anionic phosphate groups }]_{\text {nucleic acid, }}$ respectively. The resultant lipoplexes were vortexed and sonicated for 2 minutes and finally incubated at $37^{\circ} \mathrm{C}$ for 30 minutes.

\subsection{Morphology of lipoplexes}

The morphology of the resulted lipoplexes was assessed by Transmission Electron Microscopy (TEM). Briefly, $5 \varepsilon l$ of the sample were adhered onto glow discharged carbon coated grids for $60 \mathrm{~s}$. The remaining liquid was removed by blotting on a paper filter and stained with $2 \%$ uranyl acetate for 60 seconds. Samples were observed under the microscope, TECNAI G2 20 TWIN (FEI, Eindhoven, The Netherlands), operating at an accelerating voltage of $200 \mathrm{KeV}$ in a bright-field image mode. Digital images were acquired with an Olympus SIS Morada digital camera.

\subsection{Electrophoretic mobility shift assay}

Antisense oligonucleotide $(0.5 \mu \mathrm{M})$ was mixed with increasing concentrations of the cationic surfactant dispersion giving rise to cationic vesicle/antisense oligonucleotide molar ratios ranging from 2 to 20 . Lipoplexes were analyzed using electrophoresis on a $20 \%$ polyacrylamide

gel at $150 \mathrm{~V}$ for 5 hours in TBE buffer 1X. Pictures were taken using Fujifilm LAS-1000 Intelligent Dark Box II using IR LAS-1000 Lite v1.2

\subsection{Zeta potential}


Zeta potential values were obtained by laser doppler velocimetry by using a Zetasizer Nano ZS (Malvern Instruments) equipped with a He-Ne red light laser $(\lambda=633 \mathrm{~nm})$. All measurement parameters and the sample preparation $(0.5 \mu \mathrm{M}$ of antisense oligonucleotide in a final volume of $50 \mu \mathrm{L})$ were carried out according to the experimental procedure described before. Studies in the presence of fetal serum bovine (FBS) were carried out by adding $5 \mu \mathrm{L}$ of FBS to the lipoplex solutions $(50 \mu \mathrm{L})$ followed by 5 minutes of incubation. In both cases, the resulting lipoplex solutions were diluted with mili-Q water to a final volume of $1 \mathrm{~mL}$. Data is shown as the average value of three runs.

\subsection{Size measurement and physical stability of vesicles}

The particle size of cationic vesicles and cationic vesicle/antisense oligonucleotide lipoplexes were determined by using a dynamic light scattering (DLS) spectrometer (LS Instruments, 3D cross correlation multiple-scattering) equipped with a He-Ne laser $(632.8 \mathrm{~nm})$ with variable intensity. Cationic surfactant vesicles were stored at $4{ }^{\circ} \mathrm{C}$ and stability studies of cationic surfactant vesicle/antisense oligonucleotide complexes were carried out by analyzing the average size of the particles at room temperature by dynamic light scattering for one month. In both cases, cationic vesicles and lipoplexes were previously sonicated and measurements were taken at a scattering angle of $90^{\circ}$, in triplicates, without diluting samples and at a constant temperature of $25^{\circ} \mathrm{C}$. The particle radius was calculated by fitting of the first cumulant parameter.

\subsection{Cell culture}

HeLa cells were cultured at $37^{\circ} \mathrm{C}, 5 \% \mathrm{CO}_{2}$ in DMEM partially supplemented with $10 \%$ fetal bovine serum, $100 \mathrm{mg} / \mathrm{mL}$ penicillin and $100 \mu \mathrm{g} / \mathrm{mL}$ streptomycin. Cells were regularly passaged in order to maintain exponential growth. Twenty-four hours before transfection at 40 to $80 \%$ confluency, cells were trypsinized and diluted 1:5 with a fresh medium without 
antibiotics (about $1-3 \times 10^{5}$ cells $/ \mathrm{mL}$ ) and transferred to a 24 -well plate (500 $\mu \mathrm{L}$ per well). Two luciferase plasmids, Renilla luciferase (pRL-TK) and Firefly luciferase (pGL3) from Promega, were used as a reporter and as a control, respectively. Renilla and Firefly luciferase vectors ( 0.1 $\mu \mathrm{g}$ and $1.0 \mu \mathrm{g}$ per well, respectively) were transfected into the cells using Lipofectamine 2000. Cells were incubated with the plasmids for 5 hours. The medium was discarded and cells were washed with PBS twice $(500 \mu \mathrm{L})$. Then, $500 \mu \mathrm{L}$ of fresh DMEM without antibiotics were added to each well. Two transfection experiments were carried out using either DMEM without FBS or DMEM supplemented with $10 \%$ of FBS. Then antisense oligonucleotides were prepared at concentrations of 20,60, 100 and $150 \mathrm{nM}$, respectively. Lipoplexes (300 $\mu \mathrm{L}$ ) (which were prepared according to the procedure described before), were previously incubated for $30 \mathrm{~min}$ at $37^{\circ} \mathrm{C}$ using HEPES buffer (20mM, pH 7.4). Later, $100 \varepsilon \mathrm{L}$ of antisense oligonucleotide and scrambled lipoplexes were added to each well. Cell lysates were prepared and analyzed twentyfour hours after transfection using the Dual-Luciferase Reporter Assay System according to the manufacturerôs protocol. Lumminiscence was measured using SpectraMax M5 spectrophotometer.

\subsection{Analysis of cell viability by the MTT colorimetric assay}

HeLa cells viability in the presence of both cationic vesicles and lipoplexes were tested at different concentrations $(20,60,100$ and $150 \mathrm{nM}$, respectively) using the MTT colorimetric assay. For each assay, cells were seeded (about $6 \times 10^{3}$ cells per well) on a 96-well plate in 200 $\mu \mathrm{L}$ of DMEM and cultured for $24 \mathrm{~h}$. Later, the culture medium was discarded and both vesicles and lipoplexes were added at gradually larger concentrations between 20 and $150 \mathrm{nM}$. Cells were incubated for $4 \mathrm{~h}$ at $37^{\circ} \mathrm{C}$ under $5 \% \mathrm{CO}_{2}$ atmosphere. The media was discarded and additional DMEM $(200 \mu \mathrm{L})$ was added. Cells were incubated for 15 hours at $37^{\circ} \mathrm{C}$. The MTT reagent was added at a final concentration of $0.5 \mathrm{mg} / \mathrm{mL}$ per $(25 \mu \mathrm{L})$ and incubated for two additional hours at $37^{\circ} \mathrm{C}$. Finally, the medium was absorbed, DMSO $(200 \mu \mathrm{L})$ and was added to 
dissolve formazan crystals (15 minutes of stirring at room temperature) and absorbance ( $\lambda=$ $570 \mathrm{~nm}$ ) was measured. Absorbance was measured using SpectraMax M5 spectrophotometer.

\section{Results}

\subsection{Morphology of the lipoplexes}

TEM pictures showed that lipoplexes at $14 / 1 \mathrm{~N} / \mathrm{P}$ ratio exhibited a discrete spherical morphology and did not aggregate under our experimental conditions (Fig. 1). Particle size of resulting lipoplexes was around $200 \mathrm{~nm}$.

\subsection{Zeta potential measurements and oligonucleotide binding capacity measurements}

Cationic surfactant vesicle/antisense oligonucleotide lipoplexes were characterized in terms of Zeta potential and oligonucleotide binding capacity. Non-ionic surfactant synthetic vesicles composed of a modified amino lipid with a double-tailed hydrocarbonated alkyl chain and polysorbate- 80 were used at several ratios ranging from 1 to 20 and using a fixed amount of antisense oligonucleotide $(0.5 \mu \mathrm{M})$ in order to determine the optimal charge ratio to form lipoplexes in serum-free conditions. As depicted in Fig. 2A, the Zeta potential displayed negative values at the lowest $\mathrm{N} / \mathrm{P}$ charge ratios $(-20 \mathrm{mV}$ for a $\mathrm{N} / \mathrm{P}$ charge ratio of 2$)$ due to the presence of the negatively charged antisense oligonucleotide. Clearly, as the concentration of cationic vesicles increased, the tendency of Zeta potential changed by decreasing this negative character in the system and thereby obtained-positive values in a range of $+12 \mathrm{mV}$ to $+20 \mathrm{mV}$ indicating an electrostatic stabilization level. The effect of fetal bovine serum (FBS) in the cationic vesicles was also evaluated (Fig. 2A). As expected, all charge ratios that were tested displayed similar behaviors in the presence of FBS. They obtained negative Zeta potential values close to $-18 \mathrm{mV}$, except for charge ratios of 14 and 16 , in which there was a slight increase that reached values of the Zeta potential between -14 and $-12 \mathrm{mV}$, respectively. 
These results clearly indicate that all charge ratios were serum-dependent in which negatively charged particles of serum were absorbed onto the surface of lipoplexes. Moreover, these results may suggest that the exact influence of serum might be dependent on the charge ratio of the cationic vesicles. Alternatively, a native electrophoretic gel assay of lipoplexes was also carried out in order to confirm at which N/P ratio oligonucleotides are complexed with our cationic surfactant vesicles. As illustrated in Fig. 2B, free DNA and complexes can be separated by native polyacrylamide gel electrophoresis. Free ASO oligonucleotide is observed in N/P charge ratios from 0 to 8 . When ASO oligonucleotide is complexed with the cationic vesicles, the resulting complexes do not enter the gel because of the size restriction. The ASO oligonucleotide was retained in wells from N/P charge ratios 10 to 18 indicating complete complexation.

\subsection{Particle size and physical stability of cationic vesicles}

Besides the characterization of lipoplexes by superficial charge and the evaluation of their binding capacity to antisense oligonucleotides, dynamic light scattering (DLS) was also used to measure the average particle size of cationic surfactant vesicle/antisense oligonucleotide complexes at charge N/P ratios of 14 and 16 along with the stability evaluation of the cationic surfactant complexes stored at $4^{\circ} \mathrm{C}$ for one month. Physical characterization of cationic surfactant vesicle/antisense oligonucleotide complexes in terms of Zeta potential and average size is displayed in Table 1. The average particle size of both N/P ratios was almost identical (ranging from 324 and $334 \mathrm{~nm}$ for charge ratios of 14 and 16, respectively). As illustrated in

Fig. 3, the average size of the complex remained practically constant for one month, according to DLS measurements.

\subsection{Cell culture}

\subsubsection{Transfection activity in serum-free conditions}


We first evaluated the transfection efficiencies at several N/P ratios $(12,14,16,18$ and 20-fold, respectively) of our cationic surfactant vesicle/antisense oligonucleotide which formed complexes in serum-free medium conditions in order to determine which charge ratio would be more effective at carrying out gene transfection experiments, as shown in Fig. 4. All selected $\mathrm{N} / \mathrm{P}$ ratios were able to silence gene expression in a range of 50 to $70 \%$ of inhibition at $60 \mathrm{nM}$. In particular, we observed good efficiencies for charge ratios of 12, 1416 and 18 obtaining similar silencing activities (63, 70, 69 and 68\% respectively) whereas lower transfection efficiencies were observed at a charge ratio of 20 which reached only $51 \%$ of luciferase inhibition. Therefore, we chose selected charge ratios of 14 and 16 to evaluate the effect of both cationic surfactant vesicles and antisense oligonucleotide forming complexes on cell viability and gene transfection, respectively.

\subsubsection{Cytotoxicity assay}

Cell viability and cytotoxicity studies were carried out (previous to cell transfection experiments) by incubating HeLa cells with cationic surfactant vesicle dispersion and lipoplexes at both charge ratios of 14 and 16, respectively. They contained different concentrations of antisense oligonucleotide $(20,60,100$ and $150 \mathrm{nM}$, respectively) in the presence of DMEM supplemented with serum (10\%) by using a tetrazolium-based colorimetric assay [29]. As depicted in Fig. 5, no significant toxicity was observed in cells compared with control samples in the presence of either cationic surfactant vesicles or lipoplexes formed with a charge ratio of 14, which obtained viabilities around $90 \%$ in all tested concentrations. HeLa cells in the presence of lipoplexes formed at a N/P charge ratio of 16 displayed a concentration-dependent toxicity which did not show any cytotoxicity at low concentrations (around 90 and $80 \%$ of cell viability at 20 and $60 \mathrm{nM}$, respectively) whereas the aforementioned lipoplexes were detrimental at high concentrations (100 and $150 \mathrm{nM})$ to cell viability (40\%) probably due to an increase of polysorbate- 80 content in the composition of the surfactant dispersion. Cell viability was also compared to positive controls by using Lipofectamine (Figure S2; Supplementary 
Part). Lipoplexes at a charge ratio of 14 along with cells in the presence of Lipofectamine did not show any cytotoxicity at the tested concentrations. Consequently, all further studies in cell culture were therefore performed at a charge ratio of 14 .

\subsubsection{In vitro transfection: Evaluation of the antisense activity}

The efficiency in the transfection of cationic surfactant vesicle/antisense oligonucleotide complexes at the optimal charge ratio of 14 was carried out at several concentrations $(20,60$, 100 and $150 \mathrm{nM}$, respectively) targeting the Renilla luciferase mRNA expression in the absence of a commercially available cationic lipids. We observed that complexes were able to mediate cellular uptake and hence exhibited high efficiencies in gene transfection at both 100 and 150 $\mathrm{nM}(83 \%$ and $87 \%$ for 100 and $150 \mathrm{nM}$, respectively). Lipoplexes were also able to silence luciferase expression at 20 and $60 \mathrm{nM}$ (4\% and $60 \%$ for 20 and $60 \mathrm{nM}$, respectively). A phosphorothioate scrambled sequence forming complexes with cationic vesicles at the same charge ratio of 14 had no effect on luciferase expression at the same tested concentrations thereby indicating the specificity of the gene knockdown experiment (Fig. 6).

The transfection efficiencies mediated by our cationic surfactant vesicles dispersion at their optimal N/P ratio were compared to commercially available cationic lipids like Lipofectamine, as a positive control, at concentrations of 20,60, 100 and $150 \mathrm{nM}$. As illustrated in Fig. 7, gene transfections carried out in the presence of Lipofectamine were more efficient only at $60 \mathrm{nM}$ (84\% versus $60 \%$ for Lipofectamine and cationic surfactant vesicles, respectively). However, both systems reached a plateau at higher concentrations (100 and $150 \mathrm{nM})$, and hence obtained similar gene knockdown activities (approximately $85 \%$ of luciferase inhibition).

\subsubsection{Effect of the fetal bovine serum (FBS) on transfection experiments}


In order to evaluate the effect of serum on cellular uptake, we studied the efficiency of cationic vesicle/antisense oligonucleotide complexes at a charge ratio of 14 to mediate cellular uptake at several FBS concentrations $(0,10,40$ and $60 \%$, respectively) at $60 \mathrm{nM}$. The results depicted in Fig. 8 showed a clear reduction in the transfection efficiency at high FBS concentrations although cellular uptake was not totally inhibited (23\% for FBS at $60 \%$ ). In contrast, while the use of lower FBS concentrations (10\%) promoted similar transfection efficiencies than in the absence of serum (from $70 \%$ to $60 \%$ of gene knockdown activity), cellular uptake experiments carried out with FBS at $40 \%$ caused a 2 -fold decrease in luciferase inhibition activity (36\% reduction) in comparison with the maximal efficiency achieved in serum-free conditions. These results demonstrated the dependency of cationic lipoplexes at their optimal N/P ratio in the presence of high serum concentration and their efficiencies on cellular transfection.

\section{Discussion}

The emergence of new therapies such as antisense technology or more recently RNA interference has allowed therapeutic oligonucleotides to be seen as promising units for fighting against several diseases because of their high specificity in the inhibition of target proteins. However, it is also well-known that delivery of nucleic acids is the major challenge because they have to cross several cellular barriers including plasma membrane. Low cellular uptake is the main bottleneck for the development of nucleic acids as therapeutics.

Consequently, the development of new drug delivery systems that may facilitate cellular uptake of nucleic acids is becoming necessary. Herein, we described a novel non-viral carrier based on the use of a formulation made up of a synthetic aminolipid and non-ionic surfactant polysorbate- 80 which were able to form vesicles and consequently were able to form complexes with antisense oligonucleotides and mediate delivery in cell culture. 
It is well-reported in literature that encapsulating oligonucleotides in lipid vesicles may turn out to be a limiting step in the development of an effective formulation. Although there are several techniques to improve the efficiency in encapsulation of oligonucleotides [30] herein, we used hydration of lipid films as the main approach for forming stable cationic vesicle/oligonucleotide complexes.

To get a direct evidence of the lipoplexes formation, we examined such lipoplexes at 14/1 N/P ratio under TEM at different magnifications Fig. 1. Adding oligonucleotides to our formulation resulted in lipoplexes which did not aggregate to our experimental conditions (Fig 1-A). These lipoplexes adopted spherical morphology, the most favorable structure conformation from an energetic point of view. Lipoplexes size was around $200 \mathrm{~nm}$, slightly smaller than the size reported by dynamic light scattering in Table $1(320 \mathrm{~nm})$. At high magnification (Fig 1B), small cavities were displayed in the lipoplexes. The heterogeneous distribution of the oligonucleotides during the spontaneously and self-assembly process might cause such cavities.

We characterized which N/P ratio between cationic vesicles and antisense oligonucleotides was the optimal way to form complexes with a range of 0 to 20 -fold. The antisense oligonucleotide was partly condensed by the synthetic aminolipid unit after adding 6-fold of cationic dispersion, according to Zeta potential and electrophoretic mobility shift assay experiments. Increasing N/P ratios from 12 to 20 , more amino groups were introduced to bind to antisense oligonucleotides and thereby lead to the formation of the expected cationic surfactant vesicle/antisense oligonucleotide complexes with an increase of surface charge reaching a plateau at a charge ratio of 20.

One of the existing milestones in gene transfection is the effect induced by negatively charged plasma proteins on the surface of cationic lipoplexes which are prone to aggregation and lead to the failure of cell transfection. Antisense oligonucleotide forming complexes in a selected range of charge ratios from 12 to 20 were evaluated to promote delivery in the absence of fetal bovine 
serum in order to know the best N/P ratio for carrying out cell transfection. All tested N/P ratios were able to impart cellular uptake, being charge ratios of 14 and 16 those which obtained the best transfection efficiencies and silencing activities than the rest of the selected N/P ratios.

In order to correctly explain our cellular uptake results, we first evaluated whether the viability of HeLa cells were not affected with the use of our cationic surfactant vesicles as well as lipoplexes using the MTT colorimetric assay. Although some surfactants are seldom used in cell culture due to their toxicity [31], cationic vesicle dispersions along with lipoplexes at the charge ratio of 14 did not induce any cytotoxicity in HeLa cells at the selected concentrations. However, in the case of using lipoplexes at the N/P ratio of 16, lipoplexes did not display any cytoxicity effect at lower concentrations (20 and $60 \mathrm{nM})$ whereas toxicity of the aforementioned lipoplexes was detrimental at higher concentrations (100 and $150 \mathrm{nM})$ which had a significant negative effect on cell growth at the same charge ratio of 16 . This was probably due to the toxic effect induced by our cationic vesicle formulation and surfactant agent proportions used [31] to form lipoplexes with antisense oligonucleotides.

Cellular uptake experiments mediated by cationic surfactant vesicle/antisense oligonucleotide complexes at their optimal N/P ratio of 14 triggered in a dose-response manner the best gene knockdown activities at 100 and $150 \mathrm{nM}$. These silencing activities were comparable when commercially available cationic lipids were also used at the same tested concentrations. These results suggest that this transfection properties mediated by our surfactant lipoplexes might be due to structural changes caused in either the morphology or phase evolution of lipoplexes and their possible ability to undergo a lamellar to non-lamellar phase transition [32].

Another factor that can limit the effectiveness of cellular uptake is the presence of negatively charged serum proteins in medium due to the tendency of these particles to interact with the cationic charged surface of vesicles that induce aggregation in the system and deactivate their internalization into cells. This serum-dependence of cationic lipoplexes causes a serious 
limitation for in vivo applications and hence reduces the efficacy of the non-viral vector in gene therapy. In order to overcome this unwanted effect, authors have described some strategies like the use of serum-resistance amino acid-based cationic lipids [16], the use of PEGylated synthetic surfactant vesicles [22], the addition of helper lipids [19] or PEGylated lipids [33]. All these modifications have shown an improvement in the potency and stability of the formulation and an increase in the cellular uptake carried out by DNA complexes. Transfection experiments mediated by our surfactant cationic vesicle/antisense oligonucleotide complexes (as expected) were dependent as FBS concentration increased. In our hands, the efficacy in the transfection process of cationic particles at the optimal N/P ratio diminished obtaining only $20 \%$ of luciferase inhibition when a high concentration of FBS (60\%) was used. In the case of using low serum levels (10 or $40 \%$, respectively) better transfection results were achieved, as was expected. These results are in accordance to the literature in which the use of non-ionic surfactant molecules such as sorbitan monoesters (Spans) or polysorbate-80 (Tween-80) to stabilize cationic formulations normally have shown poor efficiency in the presence of a high concentration of serum [34].

There is a general consensus in which endocytosis mechanism has been proposed as the major mode of action of lipoplex-mediated delivery antisense oligonucleotide $[35,36]$ although other kinds of mechanisms like fusion have been suggested as well [37]. It is also well-accepted that the type of endocytosis can also depend on the particle size [38] and the net positive charged surfaces of non-viral carriers [39] which may interact with the negative charges at the cell surface and consequently release the genetic cargo from endosomes. However, despite all efforts and studies carried out to understand how this cellular entry pathway works for nucleic acid forming complexes, knowledge of their uptake mechanism is still limited [40]. According to our results, the average size of cationic surfactant vesicle/antisense oligonucleotide complexes at their optimal charge ratios of 14 and 16 gave similar sizes of around $320 \mathrm{~nm}$. This result may suggest a receptor-mediated endocytosis through the clarthrin-mediated pathway [41] as the main entering pathway for the aforementioned cationic surfactant lipoplexes by ensuing 
membrane destabilization and leading to the release of antisense oligonucleotides and therefore efficiently inhibit gene expression [34].

\section{Conclusions}

The urge to obtain new vehicles capable of improving the delivery properties of nucleic acids is essential for the development of oligonucleotide-based therapeutics. Cationic liposomes composed of helper lipids like DOPE or cholesterol are normally used for stabilizing complexes with nucleic acids and consequently often increase their transfection potencies. In this article, we used a formulation based on a modified aminolipid containing two saturated alkyl chains and a non-ionic surfactant polysorbate- 80 in order to form stable modified cationic vesicles with antisense oligonucleotides. Lipoplexes were characterized in terms of Zeta potential and dynamic light scattering. Furthermore, the ability of these surfactant cationic vesicle/antisense oligonucleotide complexes to impart cellular uptake in cell culture was also evaluated. The results confirmed that lipoplexes at their optimal N/P ratio of 14 did not show any sign of cytotoxicity in cell culture except for lipoplexes with a charge ratio of 16 in which cell viability was compromised. Lipoplexes showed a clear serum-dependence at high serum concentrations however, the use of low serum conditions $(10 \%)$ on our modified cationic surfactant vesicles promoted comparable results in cellular uptake to those obtained with commercially available cationic lipids. We believe that the presence of the modified amino containing a double-tailed hydrocarbonated lipid chain may be considered as a starting point in order to design novel cationic lipids by modifying some elements such as headgroup or a glycerol backbone and lead to the study the subsequent structure-activity relationship for the development of new gene delivery systems.

\section{Supporting Information}


Average size measurements by Dinamic Light Scattering at a charge ratio of 16 and normalized cell viability by MTT assay.

\section{Acknowledgements}

This work is supported by the Spanish Ministry of Education (Grant CTQ2010-20541), the Generalitat de Catalunya (2009/SGR/208) and the Instituto de Salud Carlos III (CB06_01_0019). CIBER-BBN is an initiative funded by the VI National R\&D\&i Plan 20082011, Iniciativa Ingenio 2010, Consolider Program, CIBER Actions and financed by the Instituto de Salud Carlos III with assistance from the European Regional Development Fund. The authors wish to thank CIBER-BBN Research Infrastructures, in particular S. Vilchez and C. Fornaguera from the Nanostructured liquid characterization unit for the characterization and stability measurements of the lipoplexes by Dynamic Light Scattering. Technical and human support provided by SGIker (UPV/EHU) is gratefully acknowledged.

\section{References}

[1] R. Kole, A. R. Krainer, S. Altman, RNA therapeutics: beyond RNA interference and antisense oligonucleotides, Nat. Rev. Drug Discov. 11 (2012) 125-140.

[2] Y-K. Oh, T. G. Park, siRNA delivery systems for cancer treatment, Adv. Drug. Del. Rev. 61 (2009) 850-862.

[3] G. F. Deleavey, M. J. Damha, Designing chemically modified oligonucleotides for targeted gene silencing, Chem \& Bio 19 (2012) 937-954. 
[4] K. Fluiter, R. F. Mook, F. Baas, The therapeutic potential of LNA-modified siRNAs: reduction of off-targets by chemical modification of the siRNA sequence, Meth. Mol. Bio. 487 (2009) 189-203.

[5] M. Giacca, S. Zacchigna, Virus-mediated gene delivery for human gene therapy, J. Controlled Release 161 (2012) 377-388.

[6] M. Raouane, D. Desmaele, G. Urbinati, L. Massaad-Massade, P. Couvreur, Lipid conjugated oligonucleotides: a useful strategy for delivery, Bioconjugate Chem. 23 (2012) 1091-1104.

[7] S. Trabulo, A. L. Cardoso, A. M. Cardoso, C. M. Morais, A. S. Jurado, M. C. Pedroso de Lima, Cell-penetrating peptides as nucleic acid delivery systems: from biophysics to biological applications, Curr. Pharm. Design 19 (2013) 2895-2923.

[8] E. Wagner, Functional polymer conjugates for medicinal nucleic acid delivery, Adv. Polymer Sci. 247 (2012) 1-29.

[9] G. Han, P. Ghosh, M. De, V. M. Rotello, Drug and gene delivery using gold nanoparticles, NanoBiotech. 3 (2007) 40-45.

[10] G. E. Hardee, L. G. Tillman, R. S. Geary, Routes and formulations for delivery of antisense oligonucleotides, Antisense Drug Tech. (2008) 217-236.

[11] R. L. Juliano, X. Ming, O. Nakagawa, The chemistry and biology of oligonucleotide conjugates, Acc. Chem. Res. 45 (2012) 1067-1076. 
[12] J. Soutschek, A. Akinc, B. Bramlage, K. Charisse, R. Constien, M. Donoghue, S. Elbashir, A. Gick, P. Hadwiger, J. Harboth, M. John, V. Kesavan, G. Lavine, R. K. Pandey, T. Racie, K. G. Rajeev, I. Rohl, I. Toudjarska, G. Wang, S. Wuschko, D. Brumcot, V. Koteliansky, S. Limmer, M. Manoharan, H. P. Vornlocher, Therapeutic silencing of an endogenous gene by systemic administration of modified siRNAs, Nature 432 (2004) 173-178.

[13] P. L. Felgner, T. R. Gadek, M. Holm, R. Roman, H. W. Chan, M. Wenz, J. P. Northrop, G. M. Ringold, M. Danielsen, Lipofection: a highly efficient, lipid-mediated DNA-transfection procedure, Proc. Natl. Acad. Sci. 84 (1987) 7413-7417

[14] T. Azzam, J. A. Domb, Current developments in gene transfection agents, Curr. Drug Deliv. 1 (2004) 165-193.

[15] S. Audouy, G. Molema, L. de Leji, D. Hoekstra, Serum as a modulator of lipoplexmediated gene transfection: dependence of amphiphile, cell type and complex stability, J. Gene Med. 2 (2000) 465-476.

[16] L. Li, H. Song, K. Luo, B. He, Y. Nie, Y. Yang, Y. Wu, Z. Gu, Gene transfer efficacies of serum-resistant amino acids-based cationic lipids: Dependence on headgroup, lipoplex stability and cellular uptake, Int. J. Pharm. 408 (2011) 183-190.

[17] C. Marchini, M. Montani, A. Amici, H. Amentisch, M. Heinz, C. Marianecci, D. Pozzi, G. Caracciolo, Structural stability and increase in size rationalize the efficiency of lipoplexes in serum, Langmuir 25 (2009) 3013-3021.

[18] Z. Hyvoenen, S. Roenkkoe, M-R. Toppinen, I. Jaeaeskelaeinen, A. Plotniece, A, Urtti, Dioleoylphosphatidylethanolamine and PEG-lipid conjugates modify DNA delivery mediated by 1,4-dihydropyridine amphiphiles, J. Contr. Rel. 99 (2004) 177-190. 
[19] T. Takahashi, A. Harada, N. Emi, K. Kono, Preparation of efficient gene carriers using a polyamidoamine dendron-bearing lipid: improvement of serum-resistance, Bioconjugate Chem. 16 (2005) 1160-1165.

[20] L. Xu, L. Feng, R. Dong, J. Hao, S. Dong, Transfection efficiency of DNA enhanced by association with salt-free catanionic vesicles, Biomacromolecules 14 (2013) 2781-2789.

[21] R. Buchiraju, S. Nama, B. Sakala, B. Chandu, R. Babu, A. Kommu, J. Chebrolu, B. Kishore, N. Yedulapurapu, Vesicular drug delivery system ï an overview, Res. J. Pharm. Bio. Chem. Sci. 4 (2013) 462-474.

[22] Y. Huang, Y. Rao, J. Chen, V. C. Yang, W. Liang, Polysorbate cationic synthetic vesicle for gene delivery, J. Biomed. Mat. Res. Part A 96A (2011) 513-519.

[23] Y-C. Liu, A-L. M. Ny, J. Schmidt, Y. Talmon, B. Chmelka, T. C. Lee, Photo-assisted gene delivery using light-responsive cationic vesicles, Langmuir 25 (2009) 5713-5724.

[24] R. S. Dias, B. Lindman, M. G. Miguel, DNA interaction with cationic vesicles, J. Phys. Chem. B 106 (2002) 12600-12607.

[25] G. Puras, J. Zárate, M. Aguirre, A. Diaz-Tahoces, M. Avilés-Trigueros, S. Grijalvo, R. Eritja, E. Fernández, J. L. Pedraz. A novel formulation based on 2,3-di(tetradecyloxy)propan-1amine cationic lipid combined with polysorbate 80 for efficient gene delivery to the retina. Pharm Res (2014) in press.

[26] G. Puras, M. Mashal, J. Zárate, M. Aguirre, E. Ojeda, S. Grijalvo, R. Eritja, J. L. Pedraz, A novel cationic niosome formulation based on 2,3-di(tetradecyloxy)propan-1-amine cationic 
lipid, squelene and polysorbate 80 for gene delivery purposes: Transfection efficiency and intracellular trafficking, J. Control. Rel. 174 (2014) 27-36.

[27] Y. Huang, J. Chen, X. Chen, J. Gao, W. Liang, PEGylated synthetic surfactant vesicles (Niosomes): novel carriers for oligonucleotides, J. Mater. Sci.: Matter Med. 19 (2008) 607-614.

[28] G. Kokotos, R. Verger, A. Chio, Synthesis of 2-oxoamide triacylglycerol analogues and study of their inhibition effect on pancreatic and gastric lipases, Chem. Eur. J. 6 (2000) 42114217.

[29] T. Mosmann, Rapid colorimetric assay for cellular growth and survival: Application to proliferation and cytotoxic assays, J. Immun. Meth. 65 (1983) 55-63.

[30] S. C. Semple, S. K. Klimuk, T. O. Harasym, N. Dos Santos, S. M. ansell, K. F. Wong, N. Maurer, H. Stark, P. R. Cullis, M. J. Hope, P. Scherrer, Efficient encapsulation of antisense oligonucleotides in lipid vesicles using ionizable aminolipids: formation of novel small multilamellar vesicle structures, Biochim. Biophys. Acta 1510 (2001) 152-166.

[31] S. C. Owen, A. K. Doak, P. Wassam, M. S. Shoichet, B. K. Shoichet, Colloidal aggregation affects the efficacy of anticancer drugs in cell culture, ACS Chem. Bio. 7 (2012) 1429-1435.

[32] J. Smisterova, A. Wagenaar, M. C. A. Stuart, E. Polushkin, G. tem Brinke, R. Hulst, J. B. F. N. Engberts, D. Hoekstra, Molecular shape of the cationic lipid controls the structure of cationic lipid/dioleylphosphatidylethanolamine-DNA complexes and the efficiency of gene delivery, J. Bio. Chem. 276 (2001) 47615-47622.

[33] P. C. Ross, S. W. Hui, Polyethyleneglycol enhances lipoplex-cell association and lipofection, Biochim. Biophys. Acta 1421 (1999) 273-283. 
[34] Y-Z. Huang, J-Q. Giao, J-L. Chen, W-Q. Liang, Cationic liposomes modified with nonionic surfactants as effective non-viral carrier for gene transfer, Colloids Surf. B: Biointerfaces 49 (2006) 158-164.

[35] I. A. Khalil, K. Kogure, H. Akita, H. Harashima, Uptake pathways and subsequente intracelular trafficking in nonviral gene delivery, Pharm. Rev. 58 (2006) 32-45.

[36] Z. Rehman, D. Hoekstra, I. S. Zuhorn, Mechanism of polyplex- and lipoplex-mediated delivery of nucleic acids: real-time visualization of transiente membrane destabilization without endosomal lysis, ACS Nano, 7 (2013) 3767-3777.

[37] N. S. Templeton, Cationic liposomes-mediated gene delivery in vivo, Biosci. Rep. 22 (2002) 283-295.

[38] P. C. Ross, S. W. Hui, Lipoplex size is a major determinant of in vitro lipofection, Gen. Ther. 6 (1999) 651-659.

[39] A. F. Adler, K. W. Leong, Emerging links between surfasse nanotechnology and endocytosis: impacto n nonviral gene delivery, Nano Today 5 (2010) 553-569.

[40] L. Wasungu, D. Hoekstra, Cationic lipids, lipoplexes and intracellular delivery of genes, J. Contr. Rel. 116 (2006) 255-264.

[41] S. Resina, P. Prevot, A. R. Thierry, Physico-chemical characteristics of lipoplexes influence cell uptake mechanisms and transfection efficacy, Plos One 4 (2009) e6058. 
Fig. 1 TEM images of lipoplexes at 14/1 N/P ratio, original magnification 7.100x (A) and 88.000x (B).
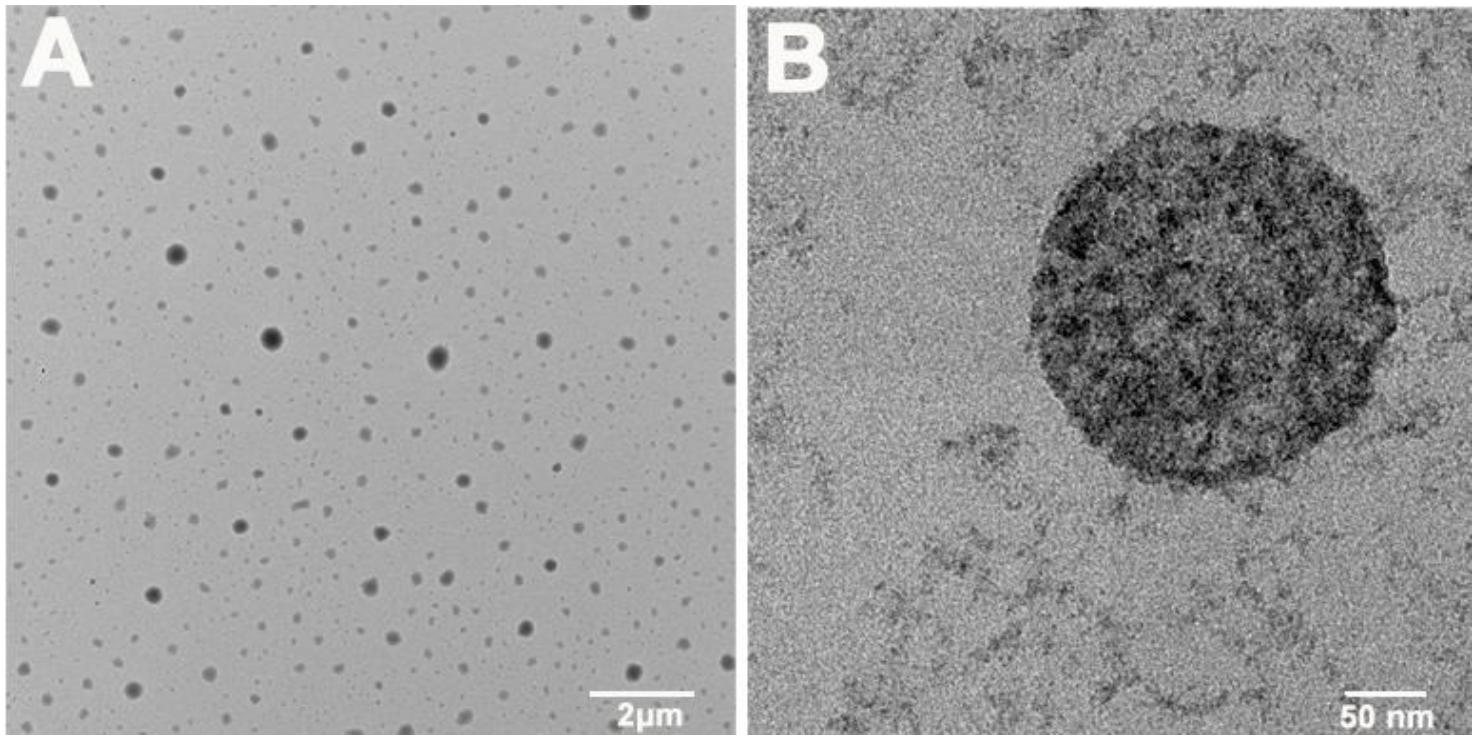
Fig. 2. Zeta potential of cationic vesicle/antisense oligonucleotide (ASO) lipoplexes at several $\mathrm{N} / \mathrm{P}$ charge ratios in the absence (circles) and the presence (triangles) of fetal bovine serum (FBS) (A) and the characterization of lipoplexes by electrophoretic mobility shift assay (B).

A

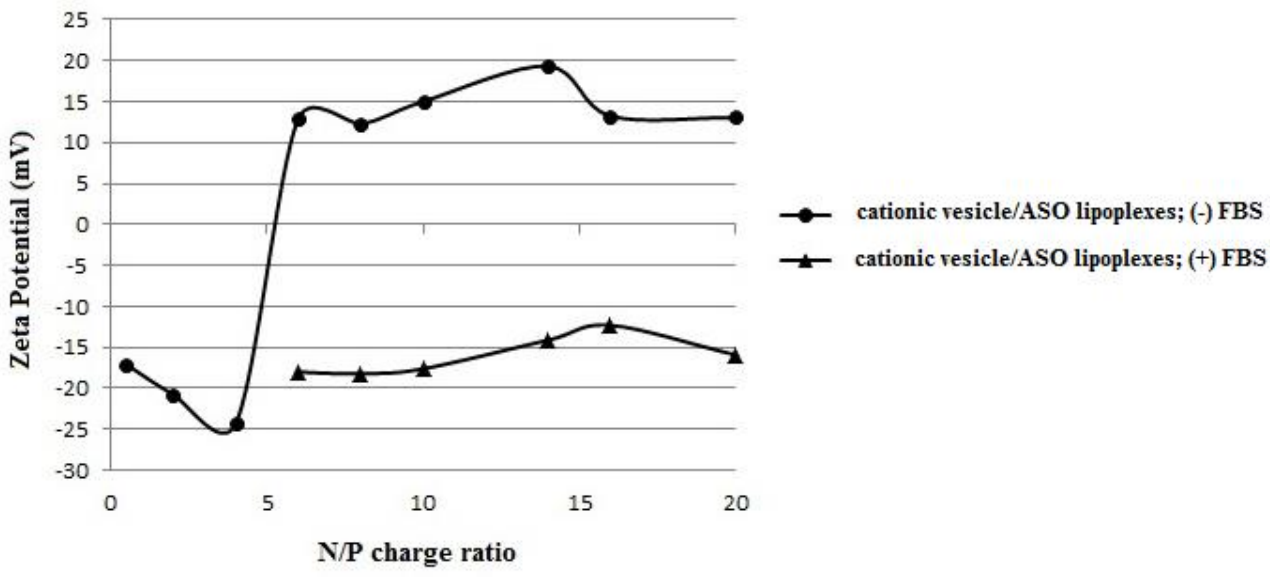

B

cationic resicles

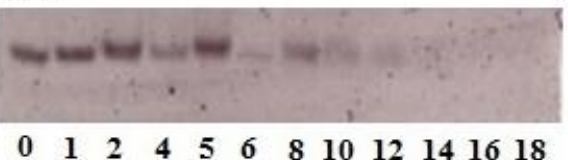

$\begin{array}{llllllllllll}0 & 1 & 2 & 4 & 5 & 6 & 8 & 10 & 12 & 14 & 16 & 18\end{array}$

$\mathrm{N} / \mathrm{P}$ charge ratio of lipoplexes 


\section{Table 1}

Physical characterization (size and Zeta potential) of cationic vesicle lipoplexes containing an antisense oligonucleotide at N/P charge ratios of 14 and 16. Results are means \pm S.D. for three independent experiments.

Cationic surfactant vesicle / antisense oligonucleotide complexes

\begin{tabular}{ccc}
\hline N/P charge ratio & 14 & 16 \\
\hline size $(\mathrm{nm})$ & $324 \pm 32.0$ & $332 \pm 3.0$ \\
6 potential $(\mathrm{mV})$ & $19.3 \pm 0.61$ & $13.2 \pm 2.0$ \\
\hline
\end{tabular}


Fig. 3. Physical stability measurements of cationic vesicle/antisense oligonucleotide (ASO) lipoplexes at a N/P charge ratio of 14 by dynamic light scattering for one month.

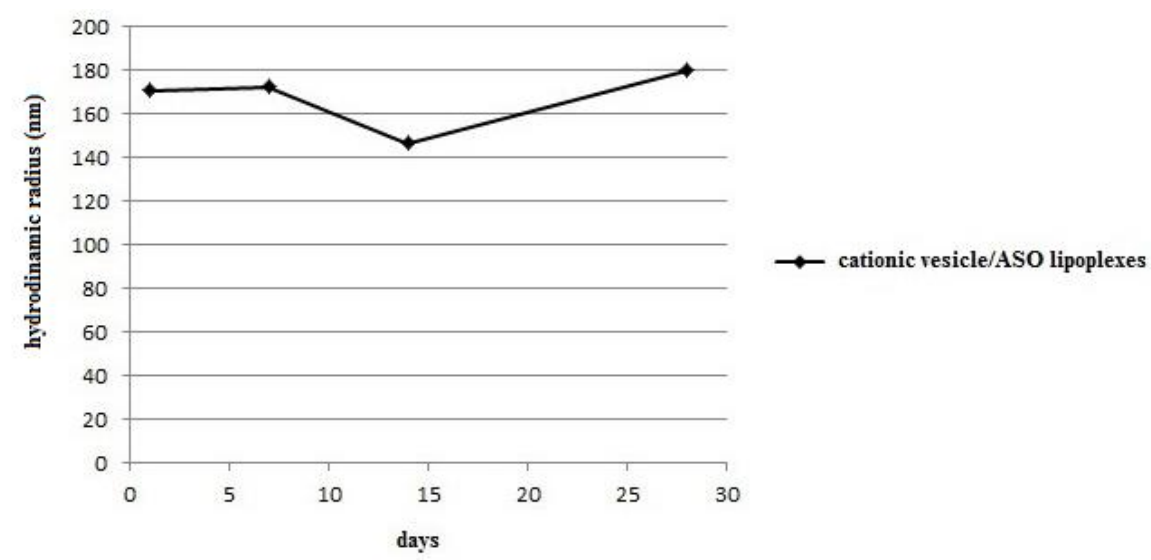


Fig. 4. Normalized transfection efficiencies mediated by cationic vesicle/antisense oligonucleotide lipoplexes targeting Renilla luciferase mRNA in serum-free conditions at several charge ratios at $60 \mathrm{nM}$. Antisense oligonucleotide (ASO) in the absence of non-viral carrier was evaluated as a control. Results are means \pm S.D. for three independent experiments.

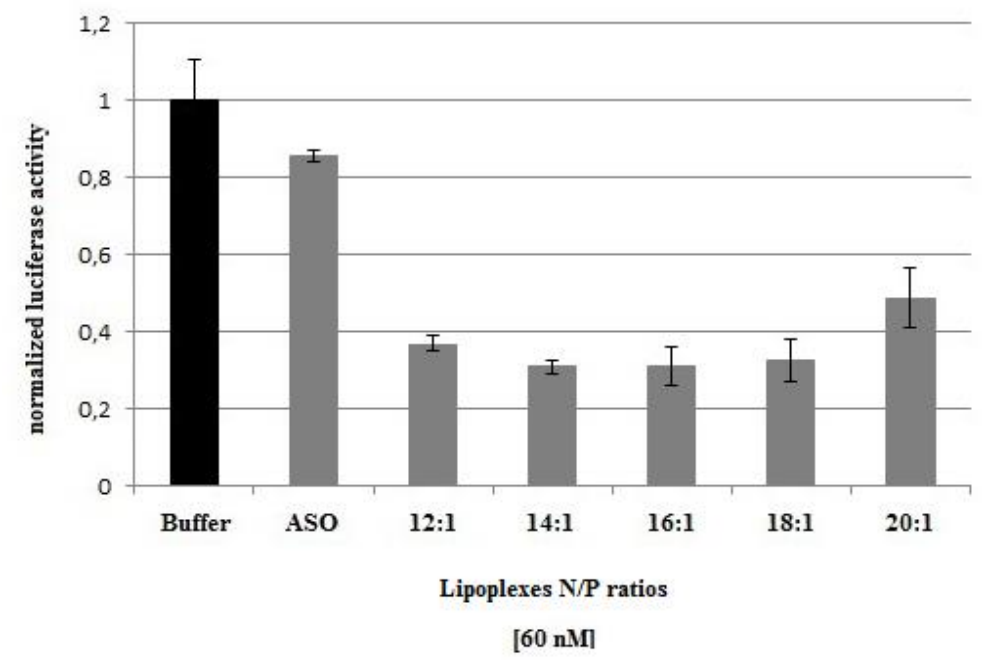


Fig. 5. Normalized cell viability of both cationic vesicle dispersions and antisense oligonucleotide forming lipoplexes at charge ratios of 14 and 16. Results are means \pm S.D. for nine independent experiments.

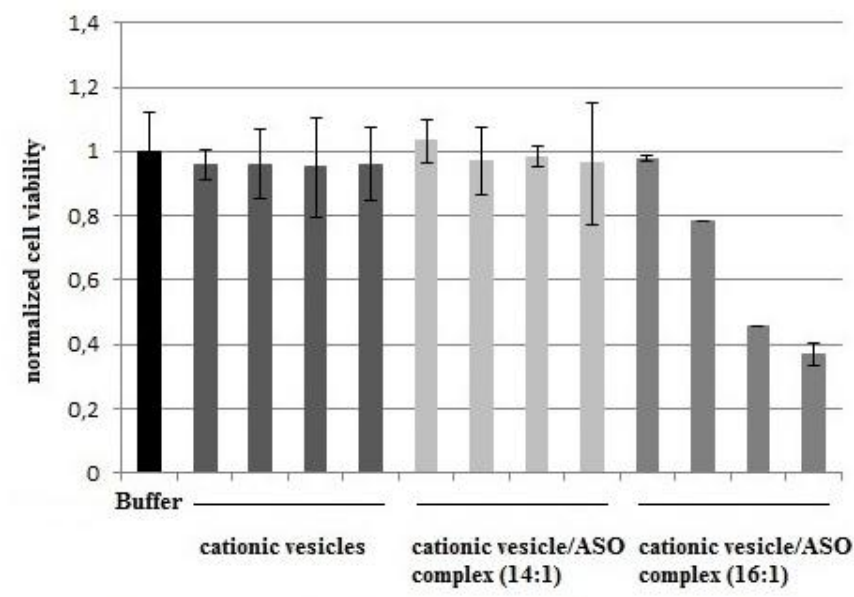

- cationic resicle dispersion

n cationic resicle/ASO oligonucleotide lipoplexes (14:1)

n cationic resicle/ASO oligonucleotide lipoplexes (16:1)

concentration [nM]

$\begin{array}{lllll}20 & 60 & 100 & 150 & 20\end{array}$ 
Fig. 6. Normalized gene-specific silencing activities targeting Renilla luciferase mRNA for cationic vesicle/antisense oligonucleotide (ASO) lipoplexes at a charge ratio of 14 at several concentrations $(20,60,100$ and $150 \mathrm{nM}$, respectively). Antisense oligonucleotide at the same tested concentrations in the absence of a non-viral carrier along with a scramble sequence (Scr) forming lipoplexes were used as controls. Results are means \pm S.D. for three independent experiments.

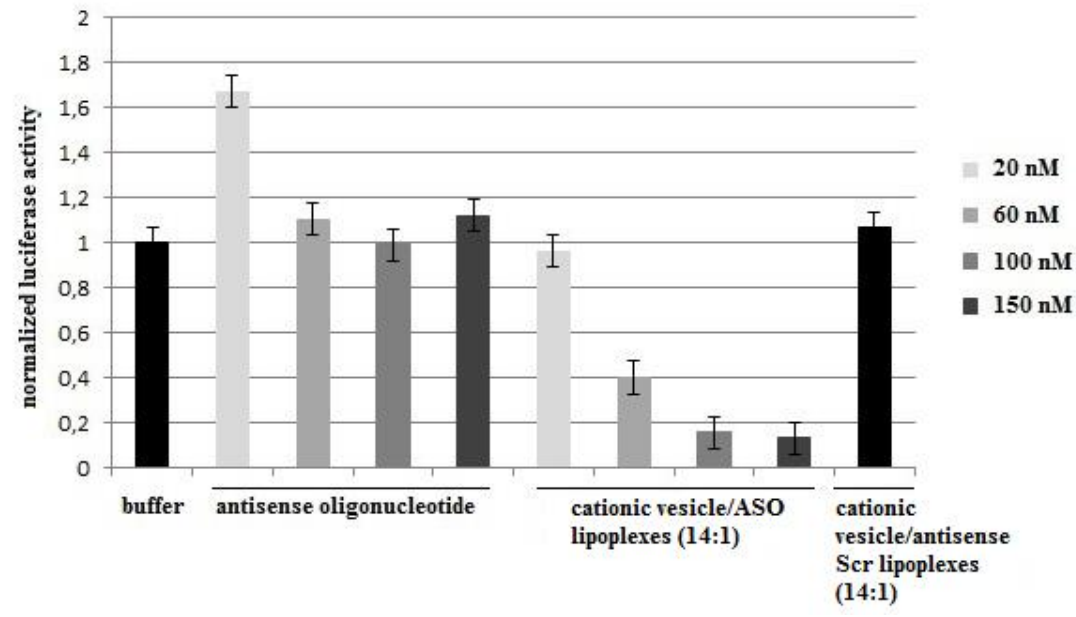


Fig. 7. Normalized gene-specific silencing activities targeting Renilla luciferase mRNA for cationic vesicles containing unmodified antisense oligonucleotide (ASO) at a N/P charge ratio of 14. Comercially available lipofectamine was used as a positive control at several concentrations $(20,60,100$ and $150 \mathrm{nM}$, respectively). Results are means \pm S.D. for three independent experiments.

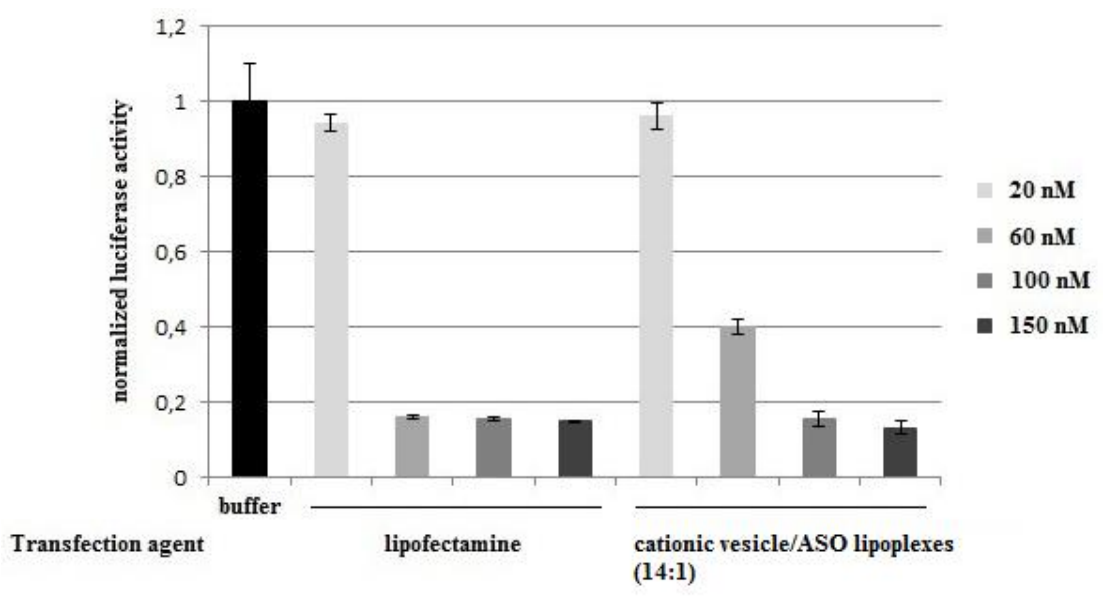


Fig. 8. Effect of fetal bovine serum (0 to 60\%) on cell transfection mediated by cationic vesicle/antisense oligonucleotide (ASO) lipoplexes at a N/P charge ratio of 14. Antisense oligonucleotide in the absence of non-viral carrier was used as a control. Results are means \pm S.D. for three independent experiments.

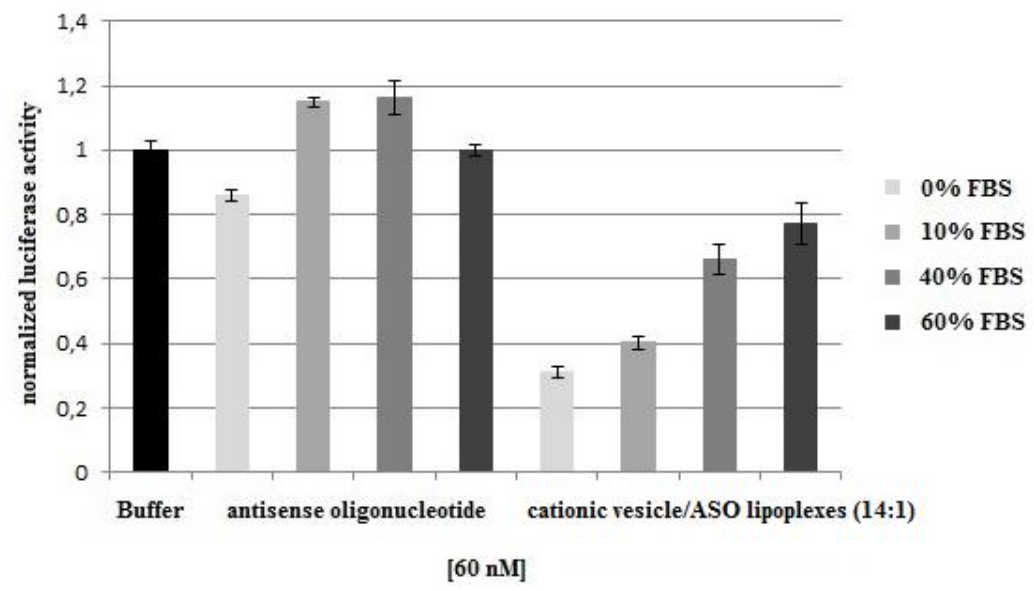

